\title{
25 Research Suare \\ Lamp1 controls CXCL10-CXCR3 axis regulated macrophage polarization and autophagy
}

Wei Gu

Bengbu Medical College https://orcid.org/0000-0002-4421-2326

Lexing Li

Huazhong Agriculture University

Xueyan Sun

Huazhong Agriculture University

Mohammad Ishaque Ali

Department of Livestock services, Dhaka

Lun Yao

Huazhong Agriculture University

Jie Liu

Southwest Minzu University

Hanchuan Dai

Huazhong Agriculture University

Guoquan Liu ( $\nabla$ guoquanliu@bbmc.edu.cn )

Huazhong Agriculture University https://orcid.org/0000-0003-3838-0590

\section{Research}

Keywords: CXCL10, CXCR3, macrophage, polarization, autophagy

Posted Date: April 21st, 2021

DOI: https://doi.org/10.21203/rs.3.rs-435551/v1

License: (c) (1) This work is licensed under a Creative Commons Attribution 4.0 International License.

Read Full License 


\section{Abstract}

\section{Background}

High expression of CXCL10 and its receptor CXCR3 is a response in inflammatory tissues and cells. Macrophage polarization and autophagy play important roles in macrophage related inflammatory. The cellular function of CXCL10-CXCR3 axis in macrophages is not well addressed. In this report, we determined the roles of CXCL10-CXCR3 axis in macrophage polarization and autophagy.

Results

First CXCL10 promotes the expression of M2 polarization markers (Arg1, Mrc1, Mmp9, VEGFa) and decreases the expression of M1 markers (IL-1b, IL-6, TNFa). In contrast, CXCR3 antagonist AMG487 promotes the expression of M1 markers and inhibits that of M2 markers. Next, we found that activation of CXCR3 by CXCL10 promotes the expression of autophagy proteins (Atg5-Atg12 complex, p62, LC3-II, and Lamp1) and AMG487 inhibits their expression. Moreover, knockdown Lamp1 by siRNA not only inhibits CXCR3 activation induced autophagy, but also switches the CXCL10-CXCR3 from M2 polarization to M1 polarization. CXCR3 activation caused the JNK expression decreased, and JNK inhibitor SP600125 treatment potentiates the CXCR3 activation induced Lamp1 expression and M2 polarization. In conclusion,

Conclusions

CXCL10-CXCR3 axis regulates the macrophage polarization and autophagy via the inhibitory JNK and Lamp1, and Lamp1 controls the switch of CXCR3 mediated M1/M2 polarization and acts as a linker between polarization and autophagy.

\section{Full Text}

Due to technical limitations, full-text HTML conversion of this manuscript could not be completed. However, the manuscript can be downloaded and accessed as a PDF.

\section{Figures}


Fig.1

A

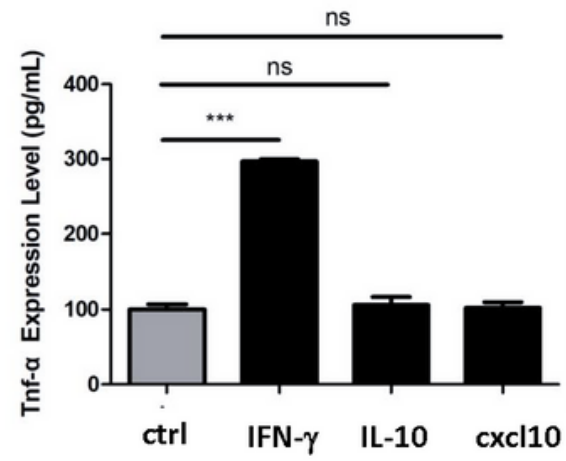

B

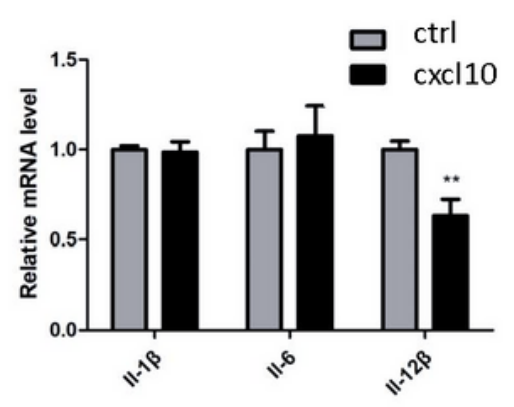

D
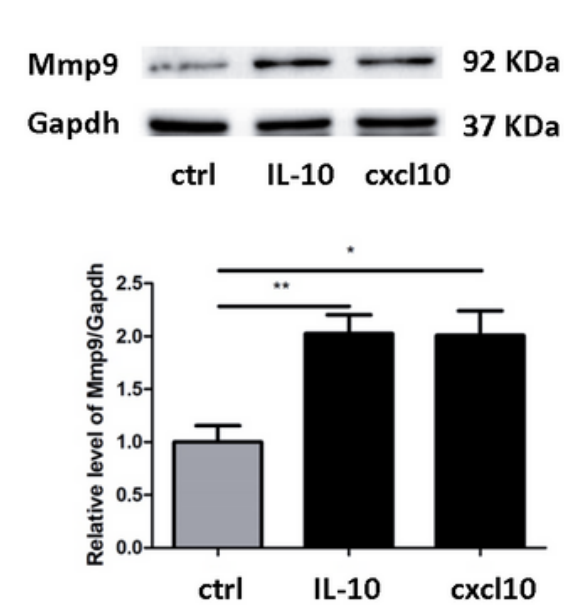

C

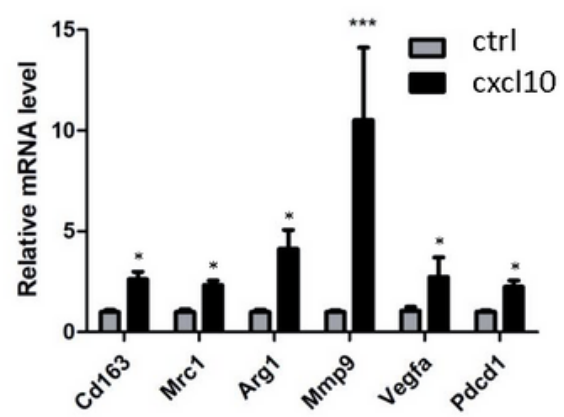

E

Pd-1

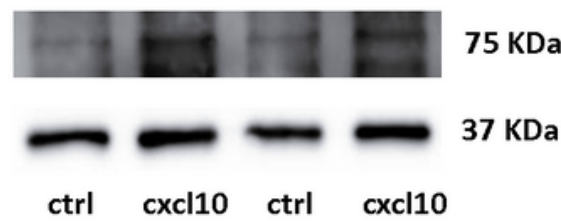

Gapdh

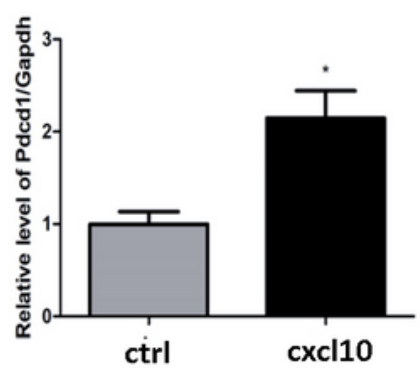

Figure 1

CXCL10 promotes M2 polarization and inhibits M1 polarization in macrophages. Cultured RAW246.7 cells were treated with INF- $\otimes(20 \mathrm{ng} / \mathrm{mL})$, IL-10 $(20 \mathrm{ng} / \mathrm{mL})$ or CXCL10 $(500 \mathrm{ng} / \mathrm{mL})$ for $24 \mathrm{~h}$, the expression of TNF囚 was measured by ELISA (A). RAW246.7 cells were treated with CXCL10 (500 ng/mL) for $24 \mathrm{~h}$, the expression of M1 markers (IL-1区, IL-6, IL-12 $\mathrm{I}$ ) (B) and M2 markers (CD163, Mrc1, Arg1, Mmp9, VEGFa, and PD-1) (C) was measured by quantitative PCR. The expression of Mmp9 (D) and PD-1(E) was 
also detected by Western blot. The data were shown in mean \pm SEM from three independent experiments. ${ }^{*} p<0.05, * \star p<0.01, * \star * p<0.005$

Fig.2
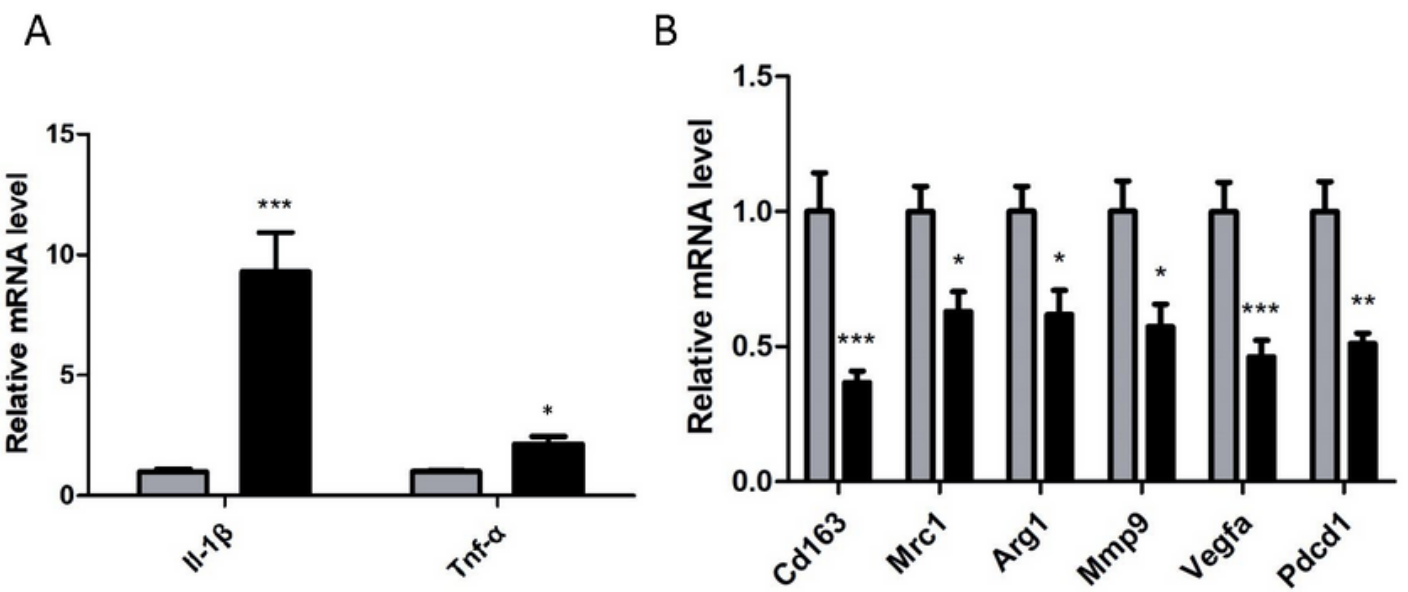

C

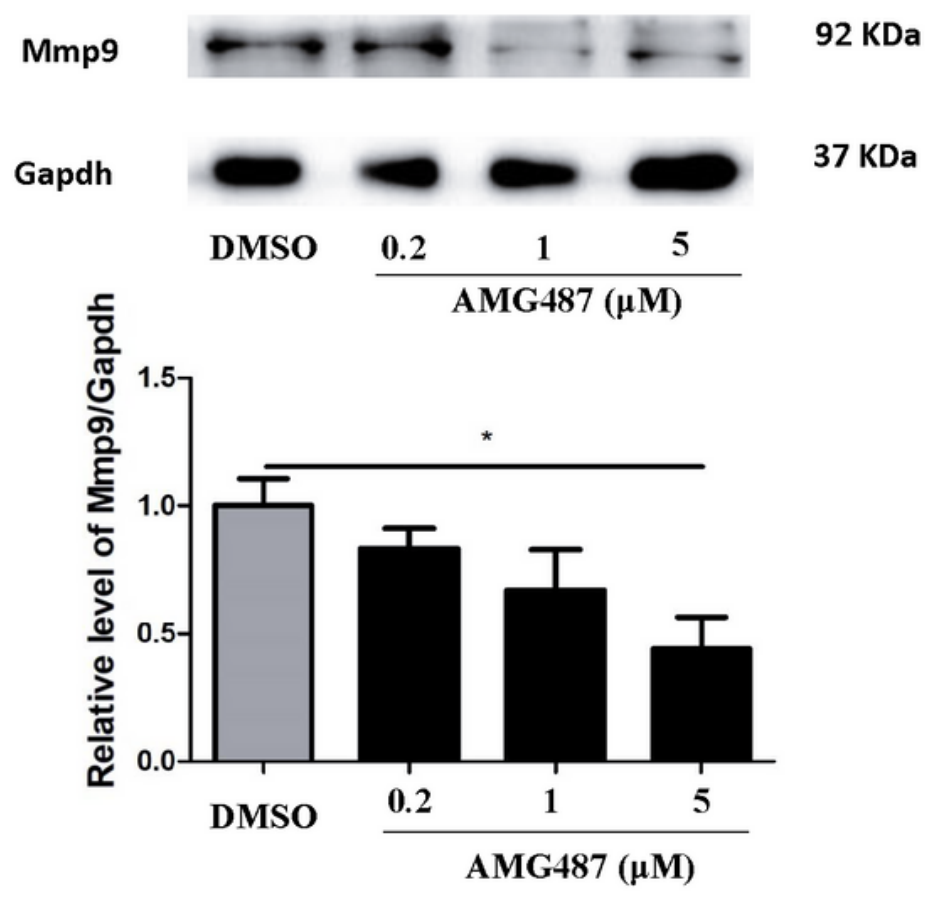

Figure 2

CXCR3 antagonist AMG487 promotes M1 polarization and inhibits M2 polarization in macrophages. Cultured RAW246.7 cells were treated with AMG487 (5 IM) for $24 \mathrm{~h}$, the expression of M1 markers (IL-1区, TNFQ) (A) and M2 markers (CD163, Mrc1, Arg1, Mmp9, VEGFa, and PD-1) (B) was measured by 
quantitative PCR. The expression of Mmp9 was also detected by Western blot (C). The data were shown

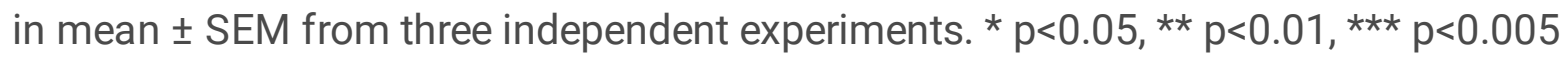

Fig.3

\begin{tabular}{|c|c|c|c|c|c|c|c|}
\hline & ctrl & cxcl10 & & & ctrl & oxcl10 & \\
\hline $\begin{array}{l}\text { Atg5-Atg12 } \\
\text { complex }\end{array}$ & $\longrightarrow$ & & $55 \mathrm{kDa}$ & Lamp1 & t & & $90-120 \mathrm{kDa}$ \\
\hline Lc3- III & 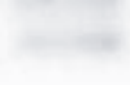 & & 14 kDa & p62 & $\longrightarrow$ & = & $60 \mathrm{kDa}$ \\
\hline Gapdh & & & $37 \mathrm{kDa}$ & Gapdh & & & $37 \mathrm{kDa}$ \\
\hline
\end{tabular}
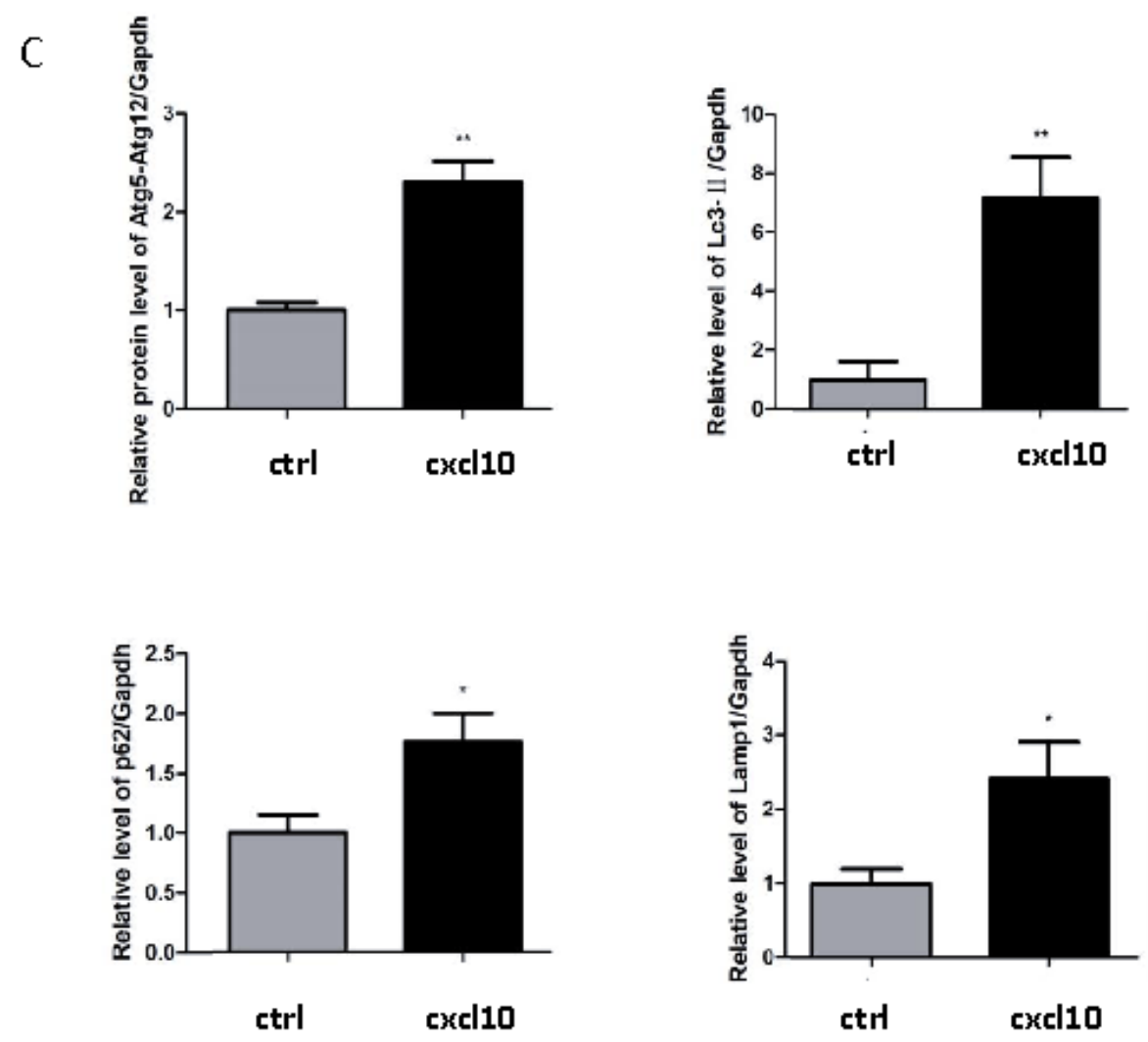

Figure 3

CXCL10 promotes autophagy in macrophages. Cultured RAW246.7 cells were treated with CXCL10 (500 $\mathrm{ng} / \mathrm{mL}$ ) for $24 \mathrm{~h}$, the expression of Atg5-Atg12 complex, Lc3-II, p62 and Lamp1 was determined by 
Western blot $(A)$. The quantitative summary was shown (B). The data were shown in mean \pm SEM from

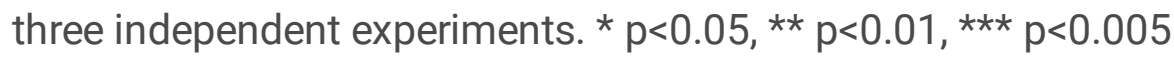

Fig.4

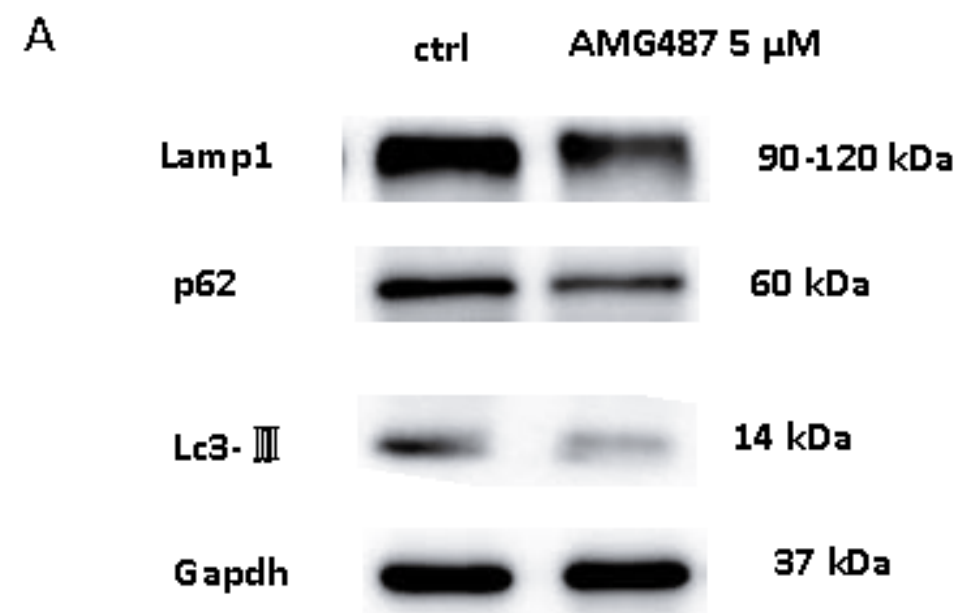

B
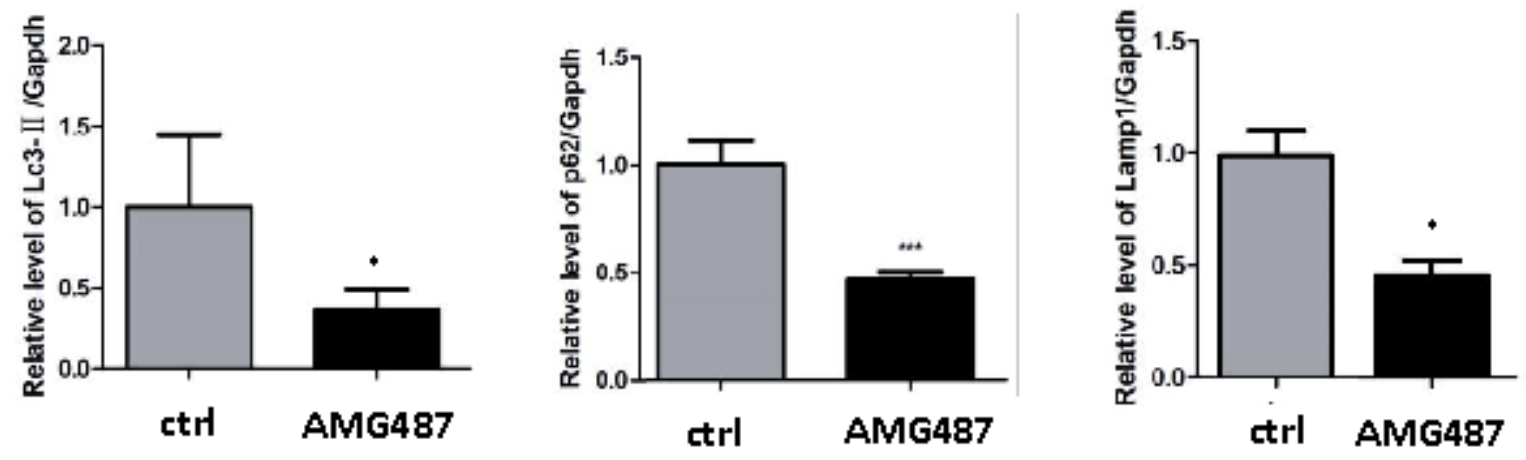

Figure 4

CXCR3 antagonist AMG487 inhibits autophagy in macrophages. Cultured RAW246.7 cells were treated with AMG487 (5 $\mathrm{\square M})$ for $24 \mathrm{~h}$, the expression of Lc3-II, p62 and Lamp1 was determined by Western blot (A). The quantitative summary was shown (B). The data were shown in mean \pm SEM from three independent experiments. ${ }^{*} p<0.05,{ }^{*} p<0.01,{ }^{* \star *} p<0.005$ 


\section{Fig.5}

A
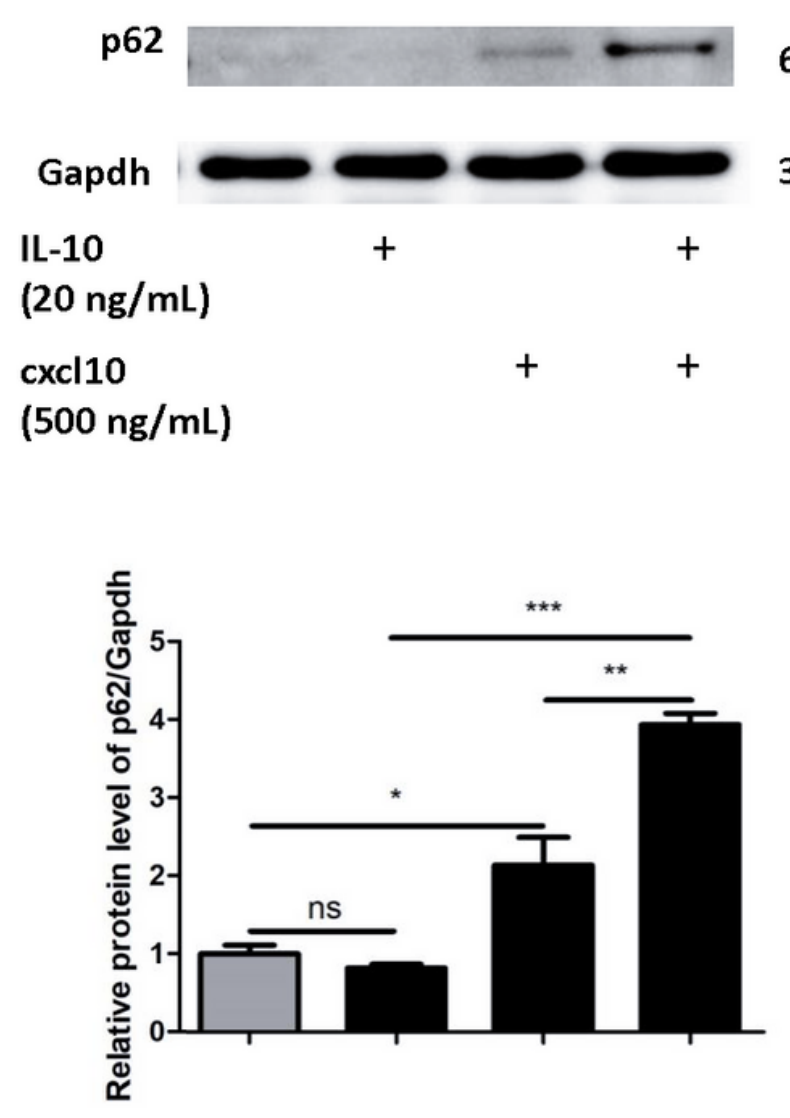

B
$14 \mathrm{kDa}$

37 kDa Gapdh

IL-10 (20 ng/mL)

cxcl10 (500 ng/mL)

\section{$37 \mathrm{kDa}$}

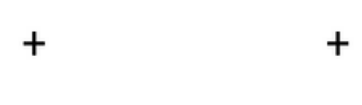

$+\quad+$

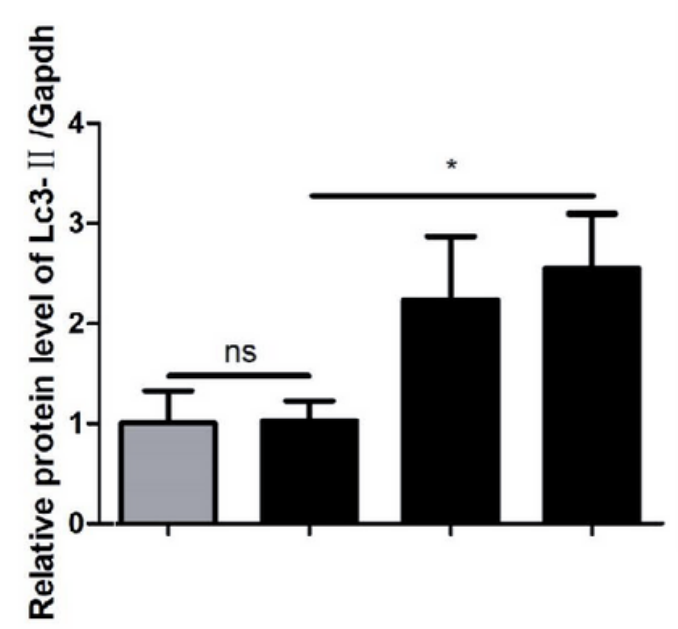

Figure 5

IL-10 potentiates the CXCL10 induced autophagy in macrophages. Cultured RAW246.7 cells were treated with IL-10 $(20 \mathrm{ng} / \mathrm{mL}), \mathrm{CXCL} 10(500 \mathrm{ng} / \mathrm{mL})$, or both for $24 \mathrm{~h}$, the expression of p62 (A) and Lc3-II (B) was determined by Western blot. The summary data were shown in mean \pm SEM from three independent experiments. ${ }^{*} p<0.05,{ }^{\star \star} p<0.01$, ${ }^{\star \star \star} p<0.005$ 
Fig. 6

A

si-NC si-Lamp

$37 \mathrm{kDa}$

90-120kDa

Lamp1

Gapdh

$C$

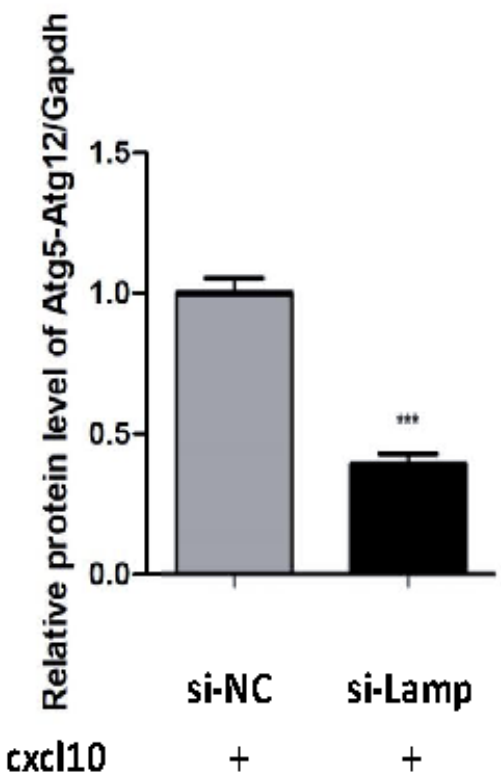

Atg5-Atg12 complex

Lt3- II

p62

Gapdh

B

$<$

-

$55 \mathrm{kDa}$

$14 \mathrm{kDa}$

$60 \mathrm{kDa}$

$37 \mathrm{kDa}$

si-NC si-Lamp

excl10 ++
$60 \mathrm{kDa}$ 
Fig.7
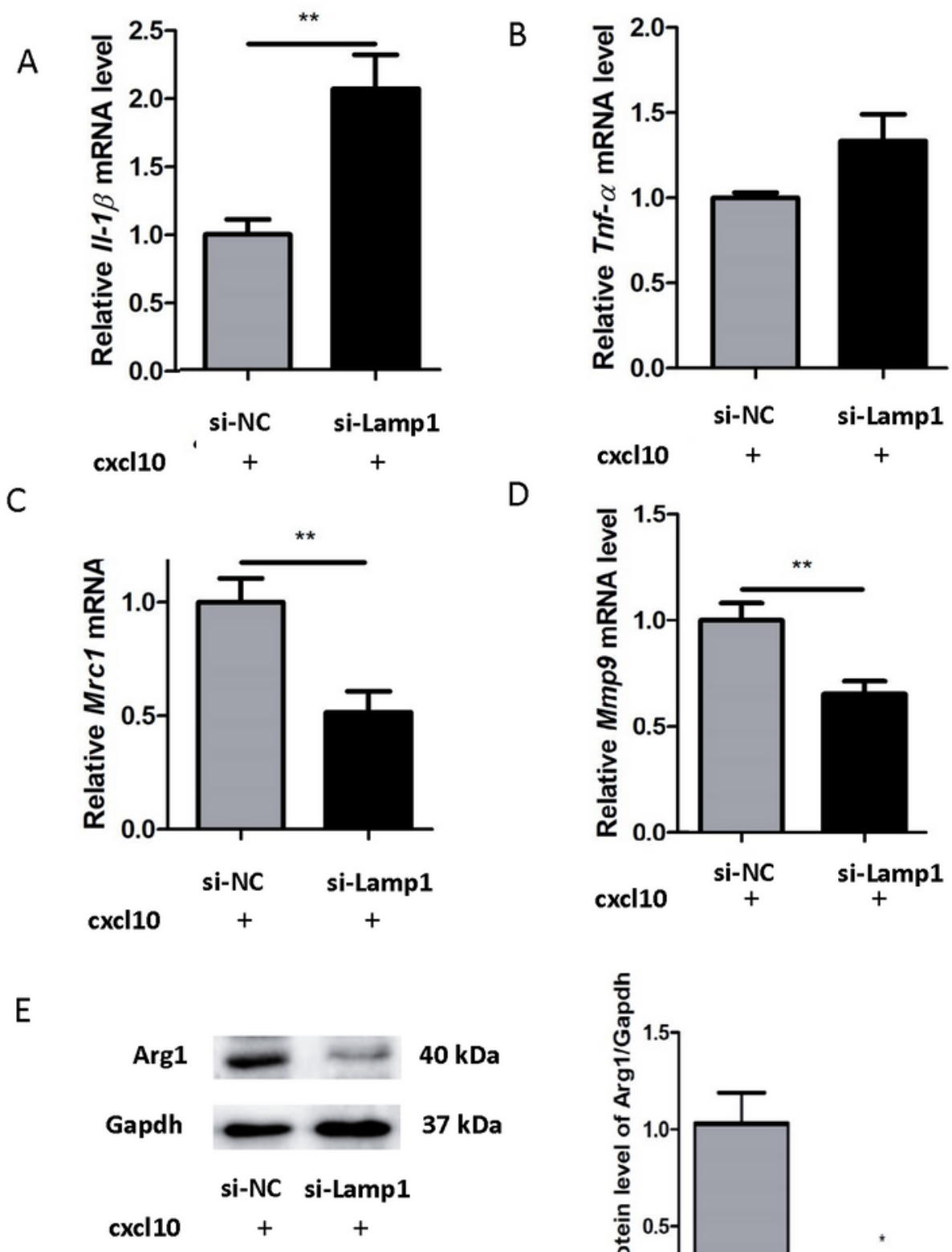

Figure 7

Knockdown of Lamp1 inhibits CXCL10 induced M2 polarization in macrophages. Cultured RAW246.7 cells were transfected with siRNA against Lamp1 then treated with CXCL10 $(500 \mathrm{ng} / \mathrm{mL})$ for $24 \mathrm{~h}$, the expression of M1 polarization markers (IL-1\ and TNFD) (A and B) and M2 polarization markers (Mrc1 and Mmp9) ( $C$ and $D$ ) was determined by quantitative PCR. The expression of M2 polarization markers 
(Arg1) was detected by Western blot (E). The summary data of Western blot and quantitative PCR were shown in mean \pm SEM from three independent experiments. ${ }^{*} p<0.05,{ }^{\star \star} p<0.01,{ }^{\star \star \star} p<0.005$

Fig.8
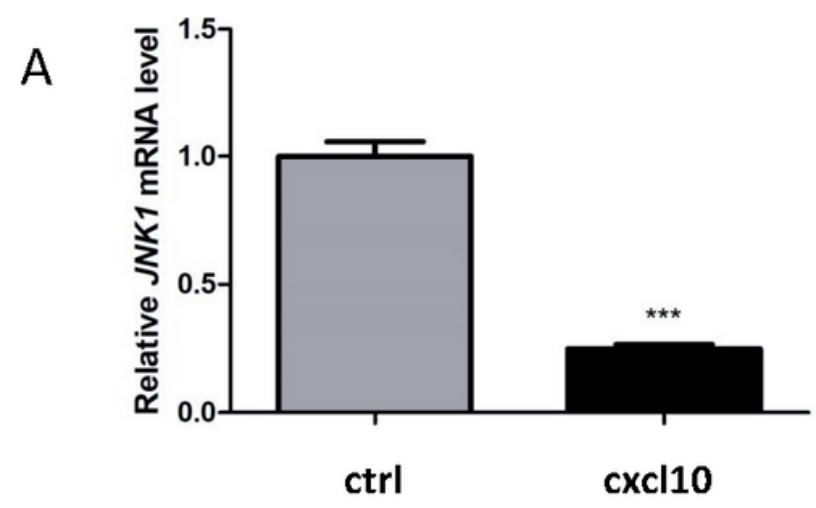

C

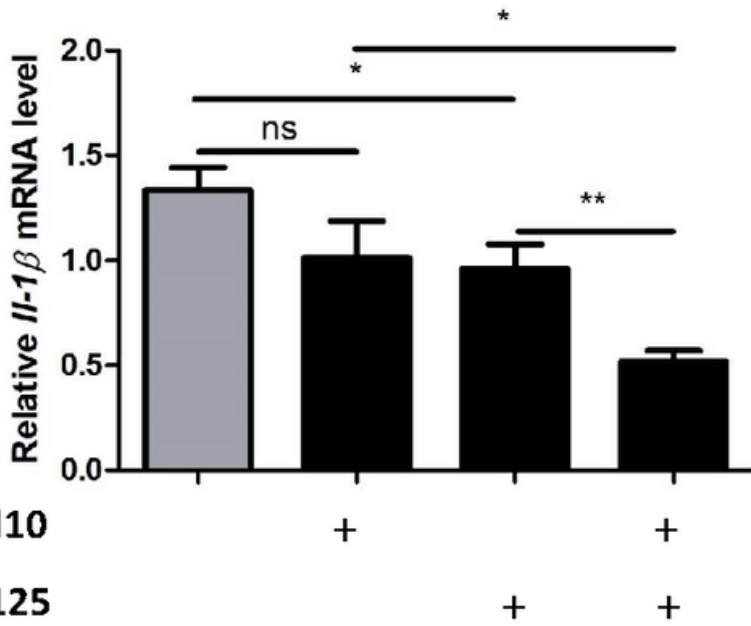

B ctrl cxcl10

JNK1 $-43 \mathrm{kDa}$

Gapdh

$37 \mathrm{kDa}$

D

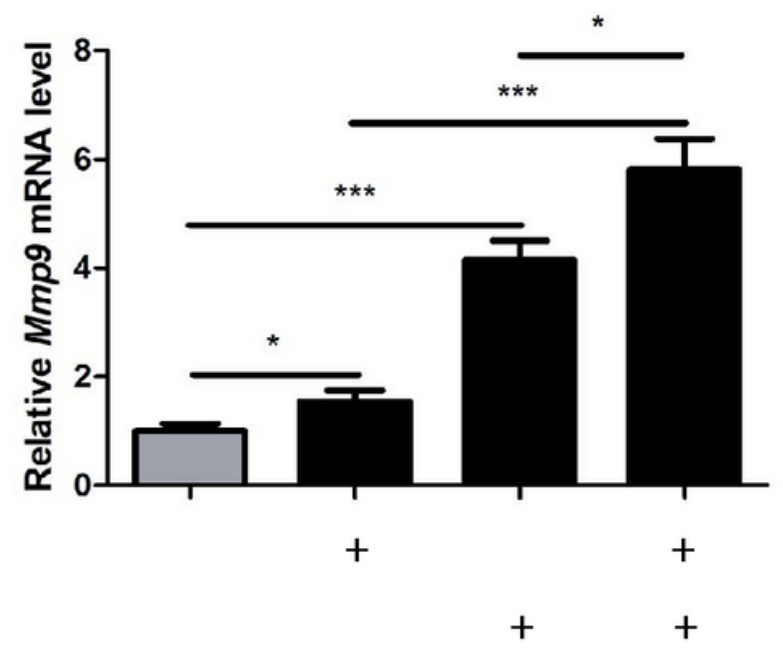

\section{Figure 8}

Inhibition of JNK potentiates CXCL10 induced M2 macrophage polarization. CXCL10 treatment decreases the expression of JNK as shown by quantitative PCR and Western blot (A). Cultured RAW246.7 cells were treated with JNK with SP600125 (50 $\mathrm{DM})$ and CXCL10 $(500 \mathrm{ng} / \mathrm{mL})$, and the expression of IL-10 (B) and Mmp9 (C) was measured with quantitative PCR. The data were shown in mean \pm SEM from three independent experiments. ${ }^{*} p<0.05,{ }^{*} p<0.01,{ }^{*} * *<0.005$ 
Fig.9

A

Lamp1

p62

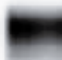

$x_{10}$
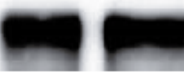

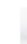

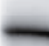

$-$

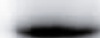

$60 \mathrm{kDa}$

Lc3-II

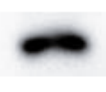

Gapdh

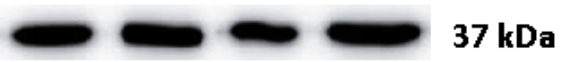

14 kDa

90-120 kDa

cxcl10

SP600125

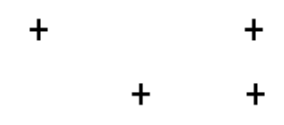

C

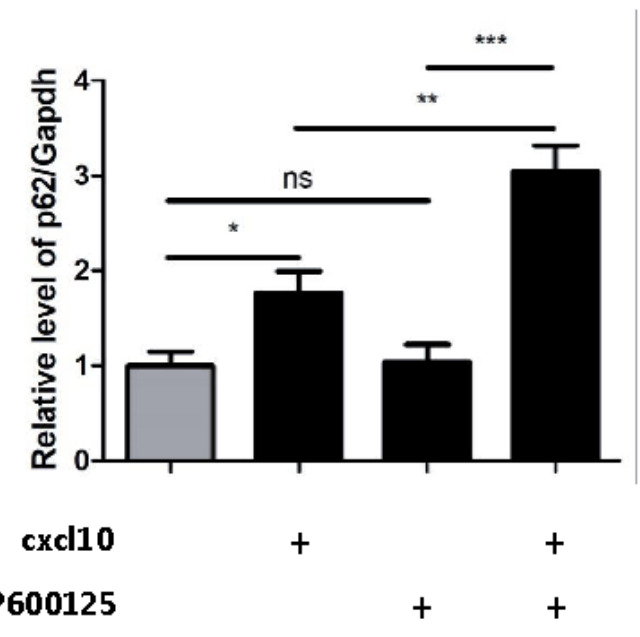

B

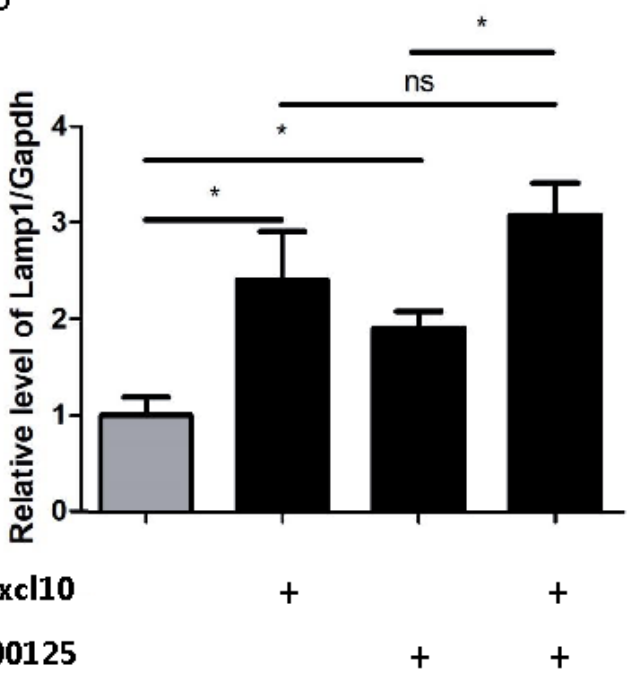

D

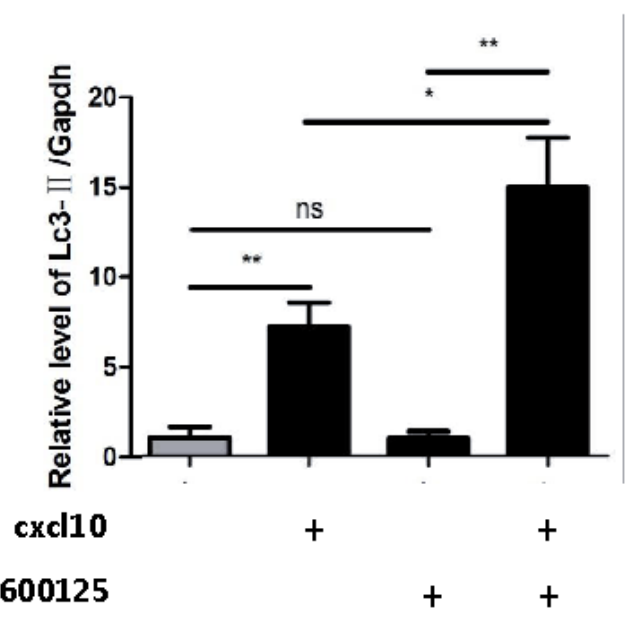

\section{Figure 9}

Inhibition of JNK potentiates CXCL10 induced autophagy in macrophages. Cultured RAW246.7 were treated JNK inhibitior SP600125 (50 घM) and CXCL10 (500 ng/mL), the expression of Lamp1, p62, and Lc3-II were detected by Western blot $(A)$. The summary data were shown in mean \pm SEM from three independent experiments. ${ }^{*} p<0.05,{ }^{* *} p<0.01,{ }^{* \star *} p<0.005$ 
Fig.10

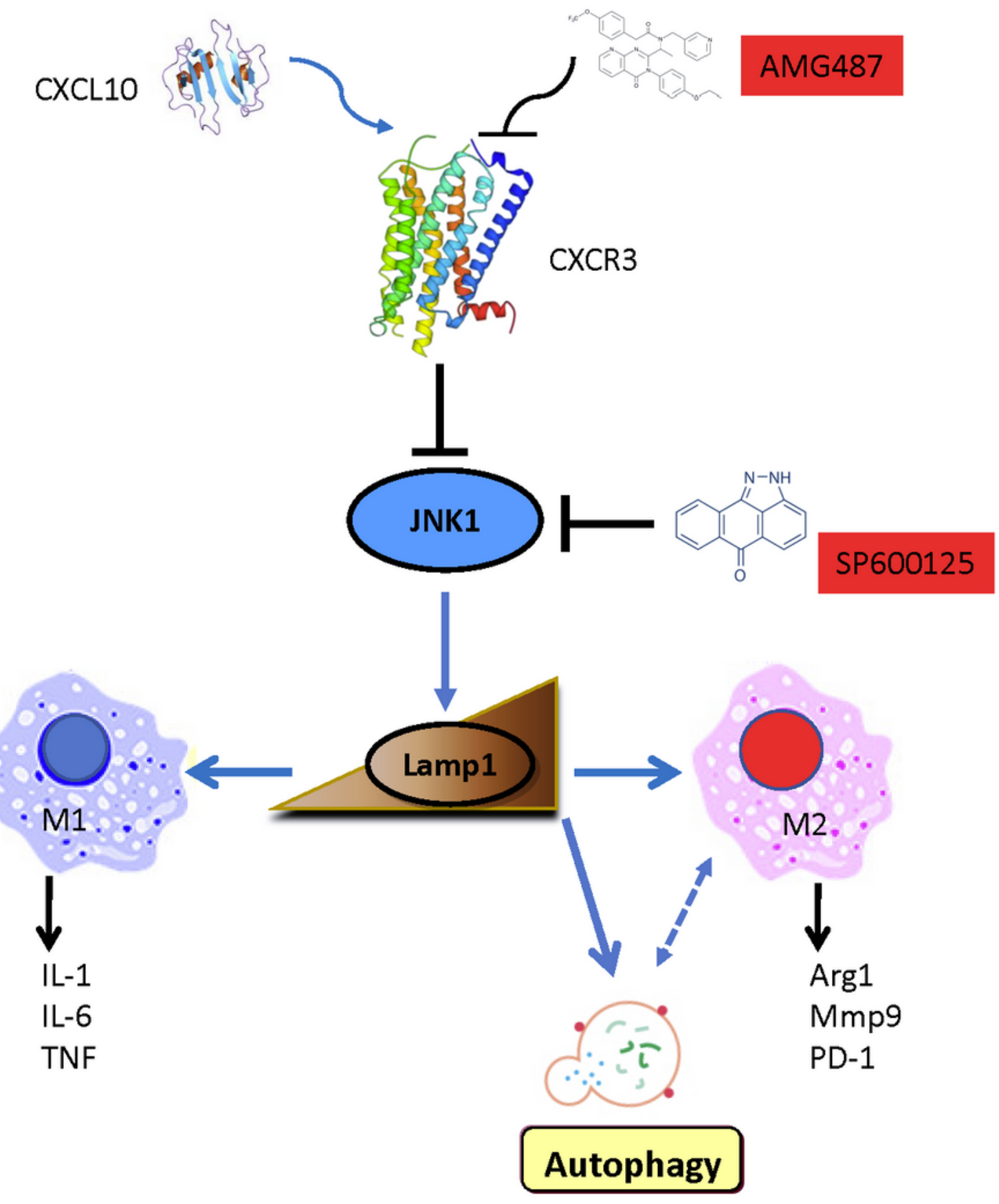

Figure 10

The summary scheme of CXCL10-CXCR3 axis in regulation macrophage polarization and autophagy.

\section{Supplementary Files}

This is a list of supplementary files associated with this preprint. Click to download. 
- $\quad$ suppl.fig.cxcr3.pdf

Page 13/13 